\title{
A new therapeutic target for advanced thyroid cancer
}

Schweppe and colleagues describe antitumor effects of a novel, oral Src inhibitor in papillary and anaplastic thyroid cancers and have also identified a potential biomarker for treatment response.

Agents designed to inhibit the mitogenactivated protein kinase pathway have had limited success in the treatment of thyroid cancer. Schweppe et al. investigated alternative signaling pathways that could provide therapeutic targets in papillary and anaplastic thyroid cancers, selecting the Src family kinases, which are involved in a number of oncogenic processes.

Schweppe's team treated five human thyroid-cancer cell lines with the Src inhibitor, AZD0530. The effects of Src inhibition were verified by means of growth and invasion assays. Treatment with AZD0530 inhibited growth and invasion in four of five thyroid-cancer cell lines; sensitivity was confirmed in two-dimensional and three-dimensional cultures.
Analysis of levels of focal adhesion kinase (FAK, a substrate of Src) and phosphorylated FAK in the thyroid-cancer cell lines and papillary thyroid tumors revealed that FAK is phosphorylated at tyrosine residue 861 (pY861) and this phosphorylation is regulated by Src. Levels of FAKpY861 were correlated with sensitivity to AZD0530, suggesting that FAK-pY861 is a potential biomarker for treatment response. AZD0530 is currently in phase II clinical trials for other tumor types, making Src inhibition a promising therapeutic strategy for patients with advanced thyroid cancer.

Lisa Richards

Original article Schweppe, R. E. et al. Inhibition of Src
with AZD0530 reveals the Src-focal adhesion kinase
complex as a novel therapeutic target in papillary and
anaplastic thyroid cancers. Eur. J. Endocrinol. doi:10.1210/
jc.2008-2511

Original article Schweppe, R. E. et al. Inhibition of Src (a) 330 reveals the Src-focal adhesion kinase i:10.1210/ jc.2008-2511 